\title{
A case of nasal mass, skin ulcers and inflammatory joint pain: granulomatosis with polyangiitis versus lymphoma
}

\author{
Authors: Panagiotis Kamperidis, ${ }^{A}$ Anthony Downs ${ }^{B}$ and Matthew Szeto ${ }^{A *}$
}

\section{Introduction}

We report a case of a 62-year-old woman presenting with a 6-month history of inflammatory joint pain in hands, knees and feet, a 3-month history of recurrent sinusitis and 'facial cellulitis', and a 2-month history of lower limb skin lesions. On examination, a soft tissue mass was eroding through the right nasal cavity. Multiple necrotic-looking ulcers were evident on her lower limbs, including on pressure points. There was mild synovitis affecting small hand and foot joints.

\section{Investigation and diagnosis}

Initial blood investigations revealed no cytopenia, but persistently raised C-reactive protein despite ongoing antibiotic treatment. Urine dipstick was negative. Magnetic resonance imaging revealed severe sinonasal mucosal thickening and a sinonasal mass with right intraorbital extension, associated with right orbital and facial cellulitis. Computed tomography (CT) with contrast of her head/sinuses revealed an enhancing, infiltrating tissue at the ethmoid air cells, nasal cavity, right orbit and nasopharynx. There was no evidence of intracranial spread. CT of the chest, abdomen and pelvis were unremarkable.

Our differential diagnoses were lymphoma, granulomatosis with polyangiitis (GPA) or IgG4 disease. Microbiology screening was negative for HIV, hepatitis B and C, and tuberculosis. The patient was also reviewed by haematology, and pictures of her skin lesions were discussed with dermatology. She was trialled on a 3-day course of pulsed intravenous methylprednisolone for likely GPA. Immunology screening ultimately revealed perinuclear anti-neutrophil cytoplasmic antibodies (pANCA) positivity, with negative myeloperoxidase (MPO), proteinase 3 (PR3) and antinuclear antibodies. The nasal mass was biopsied, and bloods were sent for immunophenotyping.

Dermatology's opinion of the skin lesion pictures was that they were consistent with necrotising vasculitis, in keeping with GPA / polyarteritis nodosa, with a characteristic necrotic centre and cherry red ulcerated peripheral rim. A skin biopsy was arranged.
However, in the meantime the nasal biopsy results were reported, revealing the diagnosis of natural killer (NK) cell lymphoma and the patient was transferred to a tertiary centre for ongoing treatment.

A case of nasal mass secondary to GPA (PR3 positive) has been previously reported by Spanuchart et al, and given the varied manifestations of the disease, GPA is an important differential to exclude in such cases. ${ }^{1}$ However, as shown in our case, mass lesions along the midline are more suggestive of lymphoma rather than vasculitis, as previously reviewed by Pakalniskis et al, and a high index of suspicion should be kept. ${ }^{2}$ Finally, perhaps more importantly, our case has highlighted the fact that cutaneous lymphoma can mimic necrotising vasculitis, so histological confirmation is strongly recommended in future similar cases.

\section{Conflicts of interest}

None declared.

\section{References}

1 Spanuchart I, Zungsontiporn N, O-charoen P, Changcharoen B, Bolger DT Jr. Granulomatosis with polyangiitis: a case of nasal mass, necrotic lung, and normal kidneys. Hawaii J Med Public Health 2015;74(9 Suppl 2):27-9.

2 Pakalniskis MG, Berg AD, Policeni BA et al. The many faces of granulomatosis with polyangiitis: a review of the head and neck imaging manifestations. AJR Am J Roentgenol 2015;205:W619-29. 